\title{
INTERPRETIVE SUMMARIES, APRIL 2011
}

Mid-infrared prediction of bovine milk fatty acids across multiple breeds, production systems, and countries. By Soyeurt et al., page $165 \%$. This study demonstrates the potential of mid-infrared spectrometry as a means to quantify the fatty acid content of bovine milk. A large data set comprising milk samples from cows of various breeds, countries, and production systems, was used in the study. Mid-infrared spectrometry is routinely used to quantify major milk components used for milk recording and milk payment. New mathematical approaches to calibrate equations for fatty acids offer the possibility of routine assessment and improvement of the quality of milk produced on farms by specific management practices and animal selection.

Contamination of cheese by polycyclic aromatic hydrocarbons in traditional smoking. Influence of the position in the smokehouse on the contamination level of smoked cheese. By Guillén et al., page 1679. Contamination of cheese by polycyclic aromatic hydrocarbons (PAH) during traditional smoking was analyzed in this study. For this purpose, the PAH profile of smoked Herreño cheese is thoroughly examined by gas chromatography-mass spectrometry, and compared with that of an unsmoked cheese. While the cheeses studied contain very few heavy PAH, the group that includes most of the carcinogenic types, they do contain high concentrations of light PAH, including alkylated compounds, whose impact on human health is not yet well established. Moreover, differences were found depending on the position of the cheeses in the smokehouse. The findings of this study could help in improving the design of smokehouses, in order to decrease the PAH contamination degree of smoked cheese.

Effect of Dioscorea opposita Thunb. (yam) supplementation on physicochemical and sensory characteristics of yogurt. By Kim et al., page 1705. Powdered yam (Dioscorea opposita Thunb.) serves as an important food source in tropical and semi-tropical countries for their nutritional and functional component benefits. The health benefits of yam include antioxidant activity, antithrombotic, anticarcinogenic, hypoglycemic, and hypolipedemic. In our study, yam powder-supplemented yogurt was found to be health beneficial due to the enrichment of two important functional components, allantoin and diosgenin. Further, these compounds do not change during pasteurization, fermentation, and storage of yogurt. Thus, yam powder-supplemented yogurt will serve as an innovative functional food in the sector of fermented food.
Improvement in melting and baking properties of low-fat Mozzarella cheese. By Wadhwani et al., page 1713. Low-fat Mozzarella cheese does not melt properly on pizza when baked because it dehydrates. By comminuting low-fat Mozzarella cheese curd in a bowl chopper and mixing with butter before pressing, low-fat Mozzarella cheese was made that had increased free oil, increased melting, and improved baking. The added butterfat was present as free fat along the curd particle junctions, as shown by laser scanning confocal microscopy, while the fat droplets present in the milk were distributed within the protein matrix of the cheese.

Short communication: Fatty acid profile of yak milk from the Qinghai-Tibetan Plateau in different seasons and for different parities. By Liu et al., page 1724. Yak milk exhibits a unique fatty acid (FA) profile and has important economic potential. Understanding the FA composition of yak milk might contribute to enhancing the quality of yak milk products. Production of milk fat with nutritionally beneficial FA profiles is influenced by grazing environment, season, and parity. However, little is known about the quality of milk from yaks kept on the Qinghai-Tibetan plateau, China. Information obtained from this study may be a promising basis for the study and exploitation of yak milk.

Hot topic: Milk and plasma disposition of thymol following intramammary administration of a phytoceutical mastitis treatment. By McPhee et al., page 1738. Little is known about the pharmacokinetics of phytoceutical products used to treat mastitis in animals on organic dairy operations. Using dairy goats as a model, this study examined the pharmacokinetics of a phytoceutical intended for intramammary treatment in dairy cattle. A gas chromatography and mass spectrometry assay was developed to detect the active chemical component thymol, in milk and plasma. Thymol was identified in both milk and plasma samples, following intramammary administration of the compound. In the future, such research will be helpful to develop withdrawal times and ensure that residues of products used to treat animals do not enter the food supply.

Relationship among seminal quality measures and field fertility of young dairy bulls using low-dose inseminations. By Christensen et al., page 1744. The relationship between bull fertility and sperm motility, morphology, viability, DNA-fragmentation, and ability of the acrosome to react was investigated 
in a large artificial insemination (AI) experiment with controlled AI dosages. Those key seminal quality traits presented a predictive value for fertility, regardless of the number of sperm per AI dose and are regarded as noncompensable sperm traits. An optimal estimation of a bull's fertility can be obtained using flow-cytometric detection of sperm concentration and viability in neat semen together with flow-cytometric detection of postthaw sperm viability.

Ex vivo phagocytic overall performance of neutrophilic granulocytes and the relation to plasma insullin-like growth factor-I concentrations in dairy cows during the transition period. By Sander et al., page 1762. An impaired function of polymorphonuclear neutrophil leukocyte function (PMNL) is reported to be one of the major reasons for increased susceptibility of dairy cows to infections during the peripartal period. In vitro studies suggest that insulin-like growth factor I (IGF-I) affects the function of PMNL. Based on the phagocytic activity, capacity, and total number of PMNL in the blood of primiparous and pluriparous cows, plasma IGF-I concentration is only a weak predictor for the phagocytotic overall performance of bovine PMNL.

Early lactation ratio of fat and protein percentage in milk is associated with health, milk production, and survival. By Toni et al., page 1772. This study estimated the predictive value of early lactation fat and protein percentage in milk for cow health, milk yield, and lactation survival. The study was conducted on 3 large Italian dairy farms. The ratio of fat-to-protein percentage in milk sampled at approximately $7 \mathrm{~d}$ postpartum was an important predictor for subsequent health events. Animals with a fat-to-protein ratio in early lactation greater than 2.0 showed an increase in postpartum diseases and an increased risk of being removed from the herd. Cows in second and higher lactation also showed a decrease in 120-day milk production with an increase of early lactation fat-toprotein ratio in milk.

Comparison of responses to Ovsynch between Holstein-Friesian and Swedish Red cows. By Keskin et al., page 1784. The Ovsynch protocol is used on many dairy farms to synchronize ovulation and allow timed artificial insemination (TAI). This study evaluated the differences between breeds in response to Ovsynch. A similar synchronization rate occurred for Holstein-Friesian and Swedish Red cows. But, the fertility (percent pregnant per TAI) at the 62-d pregnancy diagnosis was lower for Holstein-Friesian than Swedish Red. This breed difference occurred because Holstein Friesian cows had greater pregnancy loss and greater decrease in fertility during heat stress than Swedish Red cows.
Herd-level and contextual factors influencing dairy cow mortality in France in 2005 and 2006. By Raboisson et al., page 1790. Dairy cow mortality causes financial loss and is an indicator of welfare. It is an important concern for producers, policy makers, and the general public. This study reports an average mortality rate for French dairy cows. Mortality depends on both herd-level and contextual risk factors. The farmers' management style and the farming system highly influence mortality. Further investigations are needed to confirm the possible association between municipal intensification of production and low mortality.

Calving difficulty in dairy cows has a longer effect on saleable milk yield than on estimated milk production. By Barrier and Haskell, page 1804. Calving difficulty raises animal welfare and economic issues. In dairy cows, calving difficulty impaired their milk production as well as the amount of milk saleable by the farmer. However, the degree of calving difficulty from which losses were reported, their magnitude, and duration depended on the herd management. The analysis of cumulative saleable milk yields, independently of each animal having achieved a full lactation, is more representative of the income loss of the dairy producer than the cow's milk production alone. Also, it might be an indicator of the long-term biological stresses the animals experience.

Molecular identification and further characterization of Arcanobacterium pyogenes isolated from bovine mastitis and from various other origins. By Hijazin et al., page 1813. Arcanobacterium pyogenes isolated from bovine mastitis and from various other origins were identified and further characterized by polymerase chain reaction (PCR)-mediated amplification of various target genes, also including genes encoding potential virulence factors. This allowed a reliable molecular identification and an individual strain characterization and might help to elucidate the role these virulence factors play in infections caused by this bacterial pathogen.

Performance and metabolic profile of dairy cows during a lactational and deliberately induced negative energy balance with subsequent realimentation. By Gross et al., page 1820. Performance measures and concentrations of plasma metabolites were determined to study the effects of a negative energy balance (NEB) at 2 stages of lactation on adaptability in dairy cows. The extent of the change was smaller during the deliberately induced NEB by feed restriction following early lactation than during NEB in early lactation, even though the induced NEB was greater. The different effects of an energy deficiency at the 2 stages in lactation show that metabolic problems in early lactating dairy cows are not only due to an NEB. 
A descriptive study of the survival of HolsteinFriesian heifers through to third calving on English dairy farms. By Brickell and Wathes, page 1831. Low survival rates are a significant economic cost to the dairy industry. This study reports survival times and culling reasons in dairy heifers on 18 farms in the United Kingdom. Of heifers recruited at 1 mo, 11\% did not survive until first calving, $19 \%$ were culled in lactation 1 (with $24 \%$ of total days alive producing milk), and $24 \%$ were culled during lactation 2 (with $40 \%$ of days alive producing milk). Only $55 \%$ of heifers survived until third calving. Increasing the survival of individual animals would enable more cows to fulfill their genetic production potential, benefiting both profitability and welfare.

Coordination of lipid droplet-associated proteins during the transition period of Holstein dairy cows. By Koltes and Spurlock, page 1839. With the onset of lactation, many cows do not consume enough energy to meet their requirement, causing them to experience negative energy balance. Consequently, energy is mobilized from body energy reserves through the process of lipolysis. Appropriate regulation of lipolysis is critical for dairy cows to maintain health and productivity during negative energy balance. This research investigates changes in proteins involved in the regulation of lipolysis, and how these changes relate to energy balance in the transition dairy cow. Our results reveal potentially novel points of regulation of lipolysis, including associations between lipolytic proteins and energy balance-related traits.

Effect of management practices on paratuberculosis test-prevalence in Danish dairy herds. By Nielsen and Toft, page 1849. The Danish control program on paratuberculosis focuses on frequent testing with separation of animals into different risk groups, along with guidelines for handling high-risk animals. Farmers were asked to state their practices regarding paratuberculosis management, and their responses were related to decreases in test-prevalences. Closed herd management and culling of repeatedly antibody-positive cows were associated with the largest decreases in testprevalences over a 4.25 -yr period after implementation of changes in management practices.

Reduced test-day milk fat percentage in cows diagnosed with claw horn lesions during routine claw trimming. By van Straten et al., page 1858. Cows diagnosed by the professional claw trimmer with claw horn lesions during routine trimming had a lower milk fat percentage on test-days occurring 2 and 5 mo preceding the date of claw trimming. These findings support the hypothesis that subacute ruminal acidosis is associated with laminitis. Furthermore, our findings indicate that test-day milk fat percentage might be used for identifying cows and herds at risk for laminitis.

Factors associated with the rectal temperature of Holstein dairy cows during the first 10 days in milk. By Wenz et al., page 1864. Daily evaluation of the rectal temperature of postpartum dairy cows is commonly performed to facilitate the early identification and management of disease, particularly uterine infection. This study evaluated factors associated with the rectal temperature of dairy cattle during the first $10 \mathrm{~d}$ in milk. The normal rectal temperature of healthy postpartum dairy cows is greater than that generically reported for dairy cattle and is higher in primiparous than multiparous cows. This study provided a better understanding of factors associated with postpartum rectal temperature and the results will aid interpretation of rectal temperature and its effective use for managing postpartum dairy cows.

Decision tree analysis of treatment strategies for mild and moderate cases of clinical mastitis occurring in early lactation. By Pinzón-Sánchez et al., page 1873. A unique decision tree was developed to evaluate the economic impact of several treatment strategies for mild and moderate cases of clinical mastitis. Pathogen-specific losses were evaluated using several herd level distributions of causative pathogens. The results of this study can directly benefit dairy producers when making strategic treatment decisions.

Molecular epidemiological analysis of Mycoplasma bovis isolates from the Pennsylvania Animal Diagnostic Laboratory showing genetic diversity. By Soehnlen et al., page 1893. Mycoplasma is an important, but difficult to diagnose, cause of mastitis in the United States. Mycoplasma isolates submitted to the Pennsylvania Animal Diagnostic Laboratory were characterized by species. The most common and important species found was Mycoplasma bovis. Mycoplasma bovis isolates were studied at the genetic level, using amplified fragment length polymorphism, to determine the relatedness of strains. The $M$. bovis isolates submitted to the Pennsylvania Animal Diagnostic Laboratory were genetically diverse. Amplified fragment length polymorphism may serve as a valuable tool for molecular characterization of $M$. bovis strains from the United States.

Introducing heifers to freestall housing. By von Keyserlingk et al., page 1900. The aim of this study was to describe how well Holstein dairy heifers adapt to freestall housing. In 2 experiments, we assessed the changes in feeding and lying behavior when heifers were first introduced to freestalls. Heifers spent 40 to $50 \%$ less time lying down in the $24 \mathrm{~h}$ following introduction 
into the freestall pen. Heifers spent more time standing with their front 2 hooves in the stall if a neck rail was present in the stall. Heifers spent less time feeding on the day they were switched to the head-lock barrier, but recovered on the following days. In summary, lying and feeding behavior of heifers is altered following changes in housing systems.

Short communication: Evaluation of confirmatory stains used for direct microscopic somatic cell counting of sheep milk. By Petersson et al., page 1908. Milk somatic cell count is a commonly used indicator of abnormal milk. The objective of this study was to determine whether the current nonspecific, methylene blue-based stains are the appropriate regulatory stains to use for the direct microscopic somatic cell count in sheep milk. The findings of this study support the replacement of the methylene blue-based stains with the DNA-specific stain pyronin Y-methyl green for determination of the direct microscopic somatic cell count in sheep milk.

Short communication: Influence of storage and preservation on microbiological quality of silo ovine milk. By de Garnica et al., page 1922. This study was designed to determine the effects of the most common storage and preservation conditions on the count of aerobic mesophilic, psychrotrophic, thermoduric, coliform, Escherichia coli, Streptococcus agalactiae, and Staphylococcus aureus organisms in ovine milk. Unpreserved milk stored at $4^{\circ} \mathrm{C}$ significantly increased mesophilic, psychrotrophic, and coliform organisms over time. The storage at $4^{\circ} \mathrm{C}$ of azidiol-preserved milk samples was a suitable method to maintain the initial concentration for all bacterial groups and species throughout $96 \mathrm{~h}$. Freezing significantly decreased the viability of coliforms, E. coli, Strep. agalactiae, and Staph. aureus.

Prepartum nutrient intake alters palmitate metabolism by liver slices from peripartal dairy cows. By Litherland et al., page 1928. The effect of dry period diet on palmitate (fatty acid) metabolism was tested in vitro. During the far-off dry period, cows were fed to meet, exceed, or be inadequate in energy intake relative to requirements. During the close-up period, cows were fed either ad libitum or in restricted amounts. The close-up diet had little effect on palmitate metabolism. Greater esterification rates postpartum and decreased palmitate oxidation in cows overfed during the far-off dry period are consistent with triacylglycerol accumulation postpartum. In vitro metabolic activities were correlated with fat accumulation in liver but not with ketone body concentration after calving.

Effects of a perennial ryegrass diet or total mixed ration diet offered to spring-calving Holstein-
Friesian dairy cows on methane emissions, dry matter intake, and milk production. By O'Neill et al., page 1941. This study evaluated the effect of offering a perennial ryegrass diet or a total mixed ration (TMR) diet to spring-calving Holstein-Friesian dairy cows over a 10-wk period in early lactation on methane emissions, dry matter intake, and milk production. Spring-calving cows in early lactation, consuming a high quality grass diet, produced less methane per cow, per unit of intake, and per unit of fat and protein yield than those offered a TMR diet. These data are important in the context of the focus on decreasing methane emissions from dairy farming systems.

Effects of jugular-infused lysine, methionine, and branched-chain amino acids on milk protein synthesis in high-producing dairy cows. By Appuhamy et al., page 1952. High-producing dairy cows were fed a corn silage, alfalfa hay-based diet and infused with saline, methionine plus lysine, and methionine plus lysine plus branched-chain amino acids. Supplementation of methionine plus lysine significantly increased milk protein yield and milk protein content. The addition of branched-chain amino acids to methionine plus lysine did not significantly affect milk protein synthesis, indicating that none of the branched-chain amino acids were limiting for production in these diets.

Performance of lactating dairy cows fed corn as whole plant silage and grain produced from genetically modified corn containing event DAS-59122-7 compared with a nontransgenic, near-isogenic control. By Brouk et al., page 1961. Lactating dairy cows were fed forage and grain harvested from genetically modified corn plants containing event DAS-59122-7. This modification confers plants with both resistance to western corn rootworm and tolerance to glufosinate-ammonium, the active ingredient of commonly used herbicides. Compared with cows fed the diet composed of silage and grain from its nearisogenic counterpart, cows fed the diet composed of silage and grain from the modified crop had similar rates of production of milk, milk fat, and milk protein, and efficiencies of milk production. Feeding this genetically modified crop did not alter productivity of lactating dairy cows.

Effect of quantifying peptide release on ruminal protein degradation determined using the inhibitor in vitro system. By Colombini et al., page 196\%. Oligopeptides are the initial product of protein degradation and important metabolites in the rumen; however, peptides are seldom quantified directly. Absorbance at $340 \mathrm{~nm}$ of oligopeptides after reaction with $o$-phthaldialdehyde was used with the inhibitor in vitro method to quantify the contribution of peptide formation to rate of ruminal protein degradation of several 
protein concentrates. Oligopeptides were found to be an important degradation product and not quantifying the contribution of peptides to protein degradation resulted in underestimation of rates of ruminal protein degradation.

Effect of feeding different sources of rumenprotected methionine on milk production and N-utilization in lactating dairy cows. By Chen et al., page 1978. Supplementation of lactating cows with rumen-protected methionine (Met) to improve the profile of essential amino acids in metabolizable protein will often allow the feeding of decreased dietary crude protein $(\mathrm{CP})$ without losing production, resulting in increased dietary nitrogen (N) efficiency. Seventy lactating cows were used in a continuous feeding study to determine whether the isopropyl ester of 2-hydroxy4-methylthio butanoic acid (HMBi) was equivalent to Met that was physically protected from ruminal catabolism. Relative to feeding $15.6 \% \mathrm{CP}$ without supplementation, adding HMBi to the diet increased yield of energy-corrected milk and milk content of true protein and solids-not-fat (SNF), and adding either Met source increased milk true protein content and gave trends for increased fat and SNF content in milk and fat yield. Production performance on Met-supplemented diets with $15.6 \% \mathrm{CP}$ was equal to or better than that of cows fed a $16.8 \% \mathrm{CP}$ diet, but with decreased $\mathrm{N}$ excretion. Overall, results from this trial suggested that feeding HMBi gave improved milk production and $\mathrm{N}$ utilization and was similar to feeding a physically protected source of absorbed Met.

Energy expenditure of grazing cows and cows fed grass indoors as determined by the ${ }^{13} \mathrm{C}$ bicarbonate dilution technique using an automatic blood sampling system. By Kaufmann et al., page 1989. An experiment was conducted aiming to compare the energy expenditure, physical activity, and feeding behavior of grazing dairy cows and cows fed grass of the same quality in a barn using the ${ }^{13} \mathrm{C}$ bicarbonate dilution technique combined with an automatic blood sampling system. Grazing cows expended more energy, were more active, and spent more time eating than grass-fed cows in the barn. The present study showed that energy requirements for grazing cows must be reconsidered.

Effects of group composition on the incidence of respiratory afflictions in group-housed calves after weaning. By Bach et al., page 2001. Bovine respiratory disease (BRD) is the most common health problem during the post-weaning period. Evidence exists from beef production systems that health status and origin of the calves being grouped seems to be important in determining the incidence of BRD. Most dairy enterprises raise calves in individual hutches while providing milk or milk replacer, and then move them into groups after weaning. Thus, an opportunity exists to group calves after weaning according to their history of BRD while individually housed. The current study concludes that, indeed, forming a group of calves where all animals have had a previous BRD case is advantageous because it does not severely compromise growth and health of the group formed by calves with previous BRD cases, and minimizes incidence of BRD in the groups composed by calves without a previous BRD history.

Diet fermentability influences lactational performance responses to corn distillers grains: A meta-analysis. By Hollmann et al., page 200\%. A database was generated that included results from feeding trials using corn distillers grains in lactating dairy cow diets. Meta-analysis showed that diets with increased fermentability [such as increased concentrations of corn silage, starch, corn grain, or preservation method (high moisture corn vs. dry corn)] and level of milk yield and milk fat concentration influenced the effect of corn distillers grains on lactational performance. Factors of diet fermentability and level of production must be considered when determining whether and at what dietary concentrations to include distillers grains in diets for lactating dairy cows.

Dietary protein quality and quantity affect lactational responses to corn distillers grains: A meta-analysis. By Hollmann et al., page 2022. Inclusion of corn distillers grains in rations for lactating dairy cows typically increases the concentration of corn-based protein. This requires higher crude protein (CP) concentration in the ration to supply enough essential amino acids (e.g., lysine) to meet absorbed protein requirement compared with other supplemental protein sources. Thus, dietary protein quality may limit lactational performance. Our results indicate that diets with corn distillers grains should contain about $8.5 \%$ of total dietary dry matter as CP from sources other than corn-based ingredients to maximize yields of milk and protein. This limits the amount of corn distillers grains that can be fed effectively without over-feeding CP and increasing nitrogen excretion.

Nitrogen partitioning and isotopic fractionation in dairy cows consuming diets based on a range of contrasting forages. By Cheng et al., page 2031. Optimizing the utilization of dietary nitrogen $(\mathrm{N})$ is important to maximize productivity and profitability of dairy farms. A wide range of $\mathrm{N}$ intake was recorded across 9 diverse diets used in this study and around 21 and $60 \%$ of incremental $\mathrm{N}$ was excreted in feces and urine, respectively. The use of a 60/40 mixture of cereal and red clover silages allowed the production benefits of legume silages without excessive urinary $\mathrm{N}$ losses. The 
$\mathrm{N}$ isotopic fractionation approach appears unsuited for predicting $\mathrm{N}$ utilization for diets containing different amounts of ammonia-N.

Combined effects of trans-10,cis-12 conjugated linoleic acid, propionate, and acetate on milk fat yield and composition in dairy cows. By Maxin et al., page 2051. Milk fat secretion is controlled by several nutrients that are precursors or inhibitors of milk fat synthesis in the mammary gland. This study assessed the additivity of effects on milk fat secretion of 3 of these nutrients (trans-10,cis-12-CLA, propionate, and acetate), infused either alone or together. Their effects on milk fat yield and composition were additive. This suggests that with high-concentrate diets, propionate could contribute to the milk fat decreases unaccounted for by trans-10, cis-12-CLA. Moreover, this additivity could be used to estimate total milk fat changes following dietary modifications, provided that the changes in nutrient supply can be predicted.

An immunological method to screen sex-specific proteins of bovine sperm. By Sang et al., page 2060. This study was designed to identify sex-specific antibodies against bovine sex-sorted sperm, and capture sex-specific proteins. The antisera against bovine $\mathrm{X}$ - or Y-sperm were first produced by a series of immunological approaches, and further purified by immunoneutralization. Then, the purified sera with enriched sex-specific antibodies were screened for sex-specific antibodies by immunofluorescence staining and flow cytometry. The results suggested that the purified antisera against X-sperm contained sex-specific antibodies. Subsequently, a specific-sex protein was captured by the purified rabbit antisera against X-sperm.

Identification of informative cooperator herds for progeny testing based on yield deviations. By Schierenbeck et al., page 2071. A general trend in dairy cattle breeding programs is to use only a subset of herds for progeny testing. However, a lack of efficient scientific methods exists to identify those potential cooperator herds that maximize genetic differentiation among selection candidates. In our study, we evaluated a robust method to identify informative cooperator herds based on daughter yield deviations. Such methodology can be included in the process of genetic evaluation.

Association between interleukin- 8 receptor- $\alpha$ (CXCR1) polymorphism and disease incidence, production, reproduction, and survival in Holstein cows. By Galvão et al., page 2083. Recently, single-nucleotide polymorphisms (SNP) in the neutrophil interleukin-8 (IL-8) receptor (CXCR1) were identified and cows with the genotype $\mathrm{CC}+735$ had increased incidence of subclinical mastitis. The hypothesis was that cows with this genotype could have increased incidence of clinical mastitis as well as increased incidence of uterine diseases such as retained placenta, metritis, and endometritis. Furthermore, fertility, milk production, and survival could also be affected. Nonetheless, uterine diseases were not associated with the SNP and actually, cows that had the GG genotype had greater incidence of clinical mastitis. Fertility, milk production, and survival were not associated with the SNP, although these parameters were negatively affected in cows that had mastitis.

Differential expression of ruminant ZNF496 variants: Association with quantitative trait loci affecting bovine milk concentration and fertility. By Golik et al., page 2092. A polymorphism in the intergenic region upstream of the $Z N F 496$ gene on Bos taurus chromosome 7 displayed significant populationwide linkage disequilibrium with milk protein percentage in the Israeli Holstein population. Concordance was not obtained between quantitative trait loci (QTL) status of bulls and any of the polymorphisms in the functional elements of $Z N F 496$. However, $Z N F 496$ variants were differentially expressed in cattle and sheep. Thus, the search for the mutation underlying the less frequent QTL allele, which is the economically favorable allele, may be complicated by the presence of an imprinting center.

Short communication: Enhanced clinical mastitis resistance in Holsteins with a FEZL p.Gly105(12_13) polymorphism. By Sugimoto et al., page 2103. We previously identified forebrain embryonic zinc finger-like (FEZL) as a gene influencing somatic cell score (SCS). Cows with a FEZL protein harboring a stretch of 12 glycines (p.Gly105 [12]) have fewer SCS than cows with FEZL harboring a stretch of 13 glycines (p.Gly105[13]). To evaluate the impact of $F E Z L$ on clinical mastitis incidence, we genotyped the FEZL polymorphism in 918 Holstein sires and found that in sires carrying the heterozygous p.Gly105 [12], the number of daughters affected with clinical mastitis was significantly lower than in sires homozygous for p.Gly105[13]. Therefore, selection of sires carrying the p.Gly105 [12] allele could be beneficial in the dairy industry by decreasing mastitis incidence.

Short communication: Heritability of gross feed efficiency and associations with yield, intake, residual intake, body weight, and body condition score in 11 commercial Pennsylvania tie stalls. By Vallimont et al., page 2108. Feed intake was measured on 11 Pennsylvania tie stall farms and gross efficiency was slightly to moderately heritable, whereas heritability for residual intake was not significantly different from zero. Gross feed efficiency was strongly correlated with higher yield, lower body weight, and lower 
body condition. Failure to account for body condition score when defining feed efficiency would result in cows being labeled as efficient that rapidly lose body condition in early lactation.

Noncasein nitrogen analysis of ultrafiltration and microfiltration retentate. By Zhang and Metzger, page 2118. Previous research has suggested that the standard noncasein nitrogen (NCN) method for milk overestimates the NCN content of ultrafiltration and microfiltration retentate samples. This study developed a modified method that produced overall lower NCN results than the standard method. In the modified method, we used a 50-mL centrifugal tube instead of a volumetric flask to accommodate $\mathrm{pH}$ measurement after acetic acid addition. The sample was also centrifuged $(800 \times g)$ for 10 min to facilitate filtration with a smaller pore size filter paper.

Objective estimation of body condition score by modeling cow body shape from digital images. By Azzaro et al., page 2126. Body condition score is an important indicator of changes in energy reserves in dairy cows during lactation influencing milk production, animal well-being, reproduction, and, more generally, farm productivity. This study explored the possibility to efficiently semiautomate the process of body condition scoring using commercial low-cost digital cameras.

Comparisons of housing, bedding, and cooling options for dairy calves. By Hill et al., page 2138. A well-ventilated nursery with no added heat supported more weight gain than the translucent polyethylene hutches during cooler months when calves were bedded with straw. Calves housed in the nursery bedded with straw had greater weight gain, starter intake, and fewer days with scours than calves bedded with sand and housed in the nursery or hutches. Lower airborne bacteria concentrations and lower humidity were observed in the nursery than in the hutches. Summer cooling of calves with fans improved weight gain and feed efficiency, and lowered the respiration rate of calves compared to not using fans. 